\title{
A Numerical Method for Nonlinear Singularly Perturbed Multi-Point Boundary Value Problem
}

\author{
Musa Çakır, Derya Arslan \\ Department of Mathematics, Faculty of Science, University of Yuzuncu Yil, Van, Turkey \\ Email: cakirmusa@hotmail.com, ayredlanu@gmail.com
}

Received 25 April 2016; accepted 26 June 2016; published 29 June 2016

Copyright (C) 2016 by authors and Scientific Research Publishing Inc.

This work is licensed under the Creative Commons Attribution International License (CC BY).

http://creativecommons.org/licenses/by/4.0/

(c) (i) Open Access

\section{Abstract}

We consider a uniform finite difference method for nonlinear singularly perturbed multi-point boundary value problem on Shishkin mesh. The problem is discretized using integral identities, interpolating quadrature rules, exponential basis functions and remainder terms in integral form. We show that this method is the first order convergent in the discrete maximum norm for original problem (independent of the perturbation parameter $\varepsilon$ ). To illustrate the theoretical results, we solve test problem and we also give the error distributions in the solution in Table 1 and Figures 1-3.

\section{Keywords}

Singular Perturbation, Fitted Finite Difference Method, Shishkin Mesh, Nonlocal Boundary Condition, Uniform Convergence

\section{Introduction}

In this paper we shall consider singularly perturbed multi-point nonlinear problem

$$
\begin{gathered}
-\varepsilon^{2} u^{\prime \prime}+f(x, u)=0,0 \leq x \leq 1, \\
u(0)=0 \\
k_{0} u(1)=\sum_{i=1}^{m} k_{i} u\left(s_{i}\right)+k_{m+1} \int_{0}^{1} u(x) \mathrm{d} x+d \\
f(x, u) \in C^{1}([0,1] x R), \frac{\partial f}{\partial u}(x, u) \geq \alpha>0, s_{i} \in(0,1), i=1, \cdots, m, k_{0} \geq 0
\end{gathered}
$$

How to cite this paper: Çakır, M. and Arslan, D. (2016) A Numerical Method for Nonlinear Singularly Perturbed Multi-Point Boundary Value Problem. Journal of Applied Mathematics and Physics, 4, 1143-1156. 
where, $0<\varepsilon \ll 1$ is small perturbation parameter. The solution $u(x)$ has boundary layers at $x=0, x=1$.

Singularly perturbed differential equations arise many applications such as, fluid mechanics, chemical-reactor theory, the Navier-Stokes equations of fluid flow at high Reynolds number, control theory, electrical networks, and other physical models. In recent years, singularly perturbed differential equations were studied by many authors in various fields of applied mathematics and engineering. For examples, Cziegis [1] studied the numerical solution of singularly pertürbed nonlocal problem. Cziegis [2] analyzed the difference schemes for problems with nonlocal conditions. Bakhvalov [3] investigated on optimization of methods for solving boundary-value problems in the presence of a boundary layer. Amiraliyev and Çakır [4] applied the difference method on a Shishkin mesh to the singularly perturbed three-point boundary value problem. Amiraliyev and Çakır [5] researched a uniformily convergent difference scheme for singularly perturbed problem with convective term end zeroth order reduced equation. Amiraliyev and Çakır [6] studied numerical solution of the singularly perturbed problem with nonlocal boundary condition. Amiraliyev and Duru [7] estimated a note on a parameterized singular perturbation problem. Amiraliyev and Erdoğan [8] studied uniform method for singularly perturbed delay differential equations. Amiraliyeva, Erdoğan and Amiraliyev [9] applied a uniform numerical method for dealing with a singularly perturbed delay initial value problem. Adzic and Ovcin [10] studied nonlinear spp with nonlocal boundary conditions and spectral approximation. Amiraliyev, Amiraliyeva and Kudu [11] applied a numerical treatment for singularly perturbed differential equations with integral boundary condition. Herceg [12] studied the numerical solution of a singularly perturbed nonlocal problem. Herceg [13] researched solving a nonlocal singularly perturbed problem by splines in tension. Çakır [14] studied uniform second-order difference method for a singularly perturbed three-point boundary value problem. Geng [15] applied a numerical algorithm for nonlinear multi-point boundary value problems.

In this study we present uniformly convergent difference scheme on an equidistant mesh for the numerical solution of the problem (1)-(3). The difference scheme is constructed by the method integral identities with the use exponential basis functions and interpolating quadrature rules with the weight and remainder terms in integral form [5]-[7]. In Section 2, the asymptotic estimations of the problem (1)-(3) are established. The difference scheme constructed on Shishkin mesh for numerical solution (1)-(3) is presented in Section 3 and in Section 4. We prove that the method is first-order convergent in the discrete maximum norm. In Section 5, a numerical example is considered. The results show that the uniform finite difference method on Shishkin mesh is more powerful method than other methods for nonlinear singularly perturbed multi-point boundary value problem.

\section{The Continuous Problem}

In this section, we describe some properties of the solution of (1) with Lemma 2.1. we use $\|g\|_{\infty}$ for the continuous maximum norm on the $[0,1]$, where $g(x)$ is any continuous function.

Lemma 2.1.

Let $f(x, u) \in C^{1}([0,1] x R)$ and $\frac{\partial f}{\partial u}(x, u)$ is uniformly bounded in $u(x)$. We assume that

$$
\sum_{i=1}^{m} k_{i} w_{0}\left(s_{i}\right)+k_{m+1} \int_{0}^{1} u(x) \mathrm{d} x<k_{0}
$$

where $w_{0}(x) \geq|w(x)|$,

$$
\begin{gathered}
-\varepsilon^{2} w^{\prime \prime}+a(x) w(x)=0 \\
w(0)=0, w(1)=1
\end{gathered}
$$

solution of this problem.

So, the solution of the Equation (1) satisfies the inequalities

$$
\|u(x)\|_{C[0,1]} \leq C_{0}
$$

and

$$
\left|u^{\prime}(x)\right| \leq C\left\{1+\frac{1}{\varepsilon}\left[\mathrm{e}^{\frac{-\sqrt{\alpha} x}{\varepsilon}}+\mathrm{e}^{\frac{-\sqrt{\alpha}(1-x)}{\varepsilon}}\right]\right\}, 0<x<1
$$


where, $C_{0}$ and $C$ are constants independent of $\varepsilon$.

Proof. We rewrite the Equation (1). Hence, we use intermediate value theorem for $f(x, u)$,

$$
\begin{gathered}
\frac{f(x, u)-f(x, 0)}{u-0}=\frac{\partial f}{\partial u}(x, \vartheta), \vartheta=\gamma u, 0<\gamma<1 \\
f(x, u)=f(x, 0)+u(x) \frac{\partial f}{\partial u}(x, \vartheta)=-F(x)+a(x) u(x)
\end{gathered}
$$

where $a(x), F(x)$ are sufficiently smooth on $[0,1],[0,1] x R$ and

$$
\frac{\partial f}{\partial u}(x, \vartheta)=a(x) \geq \alpha>0, \vartheta \text {-intermediate value. }
$$

Consequently, we obtain the following linear equation,

$$
-\varepsilon^{2} u^{\prime \prime}+a(x) u(x)=F(x) .
$$

Now, let $u(1)=\lambda$ according to the Equation (3).

We can write the solution of the Equation (9) as follows

$$
\begin{gathered}
u(x)=v(x)+\lambda w(x) \\
-\varepsilon^{2} v^{\prime \prime}+a(x) v(x)=F(x) \\
v(0)=0, v(1)=0
\end{gathered}
$$

where $v(x)$ is solution of the Equations (11), (12).

First, we prove the estimate $v(x)$,

$$
|v(x)| \leq|v(0)|+|v(1)|+\alpha^{-1}\|F\|_{\infty} \leq C_{1} .
$$

Second, we prove the estimate $w(x)$,

$$
|w(x)| \leq|w(0)|+|w(1)|+\alpha^{-1}\|0\|_{\infty} \leq 1 .
$$

According to the Equation (4), $\lambda$ is a finite number. Then, from the Equations (13), (14) we have the following inequality

$$
\begin{gathered}
|u(x)| \leq|v(x)|+|\lambda||w(x)| \leq C_{1}+|\lambda| \\
|u(x)| \leq C_{0}
\end{gathered}
$$

we now prove the estimate the Equation (8).

If $u^{\prime \prime}(x)$ is pulled from the Equation (9), we obtain

$$
u^{\prime \prime}(x)=\frac{-1}{\varepsilon^{2}}[F(x)-a(x) u(x)]
$$

and from the Equation (16)

$$
\left|u^{\prime \prime}(x)\right| \leq\left|\frac{1}{\varepsilon^{2}}[F(x)-a(x) u(x)]\right| \leq \frac{C}{\varepsilon^{2}} .
$$

Now, we take derivative of the Equation (9) and if it called $u^{\prime}(x)=v_{0}(x)$, the Equation (9) takes the form with boundary condition

$$
\begin{gathered}
-\varepsilon^{2} v_{0}^{\prime \prime}+a(x) v_{0}(x)=\varnothing(x) \\
u^{\prime}(0)=v_{0}(0)=0, u^{\prime}(1)=v_{0}(1)=1 .
\end{gathered}
$$

Now, we proceed with the estimation of $\varnothing(x), u^{\prime}(0), u^{\prime}(1)$, respectively, from the Equation (7)

$$
\varnothing(x)=F^{\prime}(x)-a^{\prime}(x) u(x) \leq C_{1} .
$$


We use the following relation for $g \in C^{2}[0,1]$,

$$
g^{\prime}(x)=\frac{g(\beta)-g(\alpha)}{\beta-\alpha}-\int_{\alpha}^{\beta}\left[\frac{\beta-t}{\beta-\alpha}-T_{0}(x-t)\right] g^{\prime \prime}(t) \mathrm{d} t, \alpha<x<\beta, \alpha<\beta
$$

where

$$
T_{0}(x-t)= \begin{cases}1, & x-t>0 \\ 0, & x-t<0\end{cases}
$$

the Equation (21) with the values $g(x)=u(x), \alpha=0, \beta=\varepsilon, x=0$ and from the Equations (7)-(17)

$$
\left|u^{\prime}(0)\right| \leq\left|\frac{u(\varepsilon)-u(0)}{\varepsilon}\right|+\int_{0}^{\varepsilon}\left|u^{\prime \prime}(t)\right| \mathrm{d} t \leq \frac{C}{\varepsilon} .
$$

In a similar manner, the Equation (21) with the values $g(x)=u(x), \alpha=1-\varepsilon, \beta=1, x=1$ and from the Equations (7)-(17)

$$
\left|u^{\prime}(1)\right| \leq\left|\frac{u(1)-u(1-\varepsilon)}{\varepsilon}\right|+\int_{1-\varepsilon}^{1}\left|u^{\prime \prime}(t)\right| \mathrm{d} t \leq \frac{C}{\varepsilon} .
$$

We write the solution of the Equations (18), (19) in the form,

$$
v_{0}(x)=v_{1}(x)+v_{2}(x)
$$

where $v_{1}(x), v_{2}(x)$ are respectively the solution of the following problems,

$$
\begin{gathered}
-\varepsilon^{2} v_{1}^{\prime \prime}(x)+a(x) v_{1}(x)=\varnothing(x) \\
v_{1}(0)=0, u^{\prime}(1)=v_{1}(1)=0 \\
-\varepsilon^{2} v_{2}^{\prime \prime}(x)+a(x) v_{2}(x)=0 \\
v_{2}(0)=\left|v_{0}(0)\right|, u^{\prime}(1)=v_{2}(1)=\left|v_{0}(1)\right| .
\end{gathered}
$$

According to the maximum principle in the Equations (24), (25), we can the following Barrier function,

$$
\psi_{1}(x)=\mp v_{1}(x)+\alpha^{-1}\|\varnothing(x)\|_{\infty} .
$$

This Barrier function provides the conditions of the maximum principle and

$$
v_{1}(x) \leq C .
$$

In a similar manner, according to the maximum principle in the Equation (26), we can write

$$
v_{2}(x) \leq \theta(x)
$$

where $\theta(x)$ is the solution of the following problem with constant coefficient,

$$
\begin{gathered}
-\varepsilon^{2} \theta^{\prime \prime}(x)+\alpha \theta(x)=0 \\
\theta(0)=\left|v_{0}(0)\right| \leq \frac{C}{\varepsilon}, \theta(1)=\left|v_{0}(1)\right| \leq \frac{C}{\varepsilon}
\end{gathered}
$$

where $a(x) \geq \alpha>0$ and the solution of $\theta(x)$ as follows,

$$
\theta(x)=\frac{v_{0}(0)\left[\mathrm{e}^{\frac{-\sqrt{\alpha}(1-x)}{\varepsilon}}-\mathrm{e}^{\left.\frac{\sqrt{\alpha}(1-x)}{\varepsilon}\right]}+v_{0}(1)\left[\mathrm{e}^{\frac{-\sqrt{\alpha} x}{\varepsilon}}-\mathrm{e}^{\frac{\sqrt{\alpha} x}{\varepsilon}}\right]\right.}{\left[-\mathrm{e}^{\frac{\sqrt{\alpha}}{\varepsilon}}+\mathrm{e}^{\frac{-\sqrt{\alpha}}{\varepsilon}}\right]}
$$

after some arragement, we can obtain, 


$$
\theta(x) \leq \frac{C}{\varepsilon}\left[\mathrm{e}^{-\frac{\sqrt{\alpha} x}{\varepsilon}}+\mathrm{e}^{\frac{-\sqrt{\alpha}(1-x)}{\varepsilon}}\right] .
$$

Finally, from $u^{\prime}(x)=v_{0}(x),\left|v_{1}(x)\right| \leq C, \theta(x) \leq v_{2}(x), v_{0}(x)=v_{1}(x)+v_{2}(x)$, we have the following inequality,

$$
\begin{aligned}
\left|u^{\prime}(x)\right| & =\left|v_{0}(x)\right| \leq\left|v_{1}(x)\right|+\left|v_{2}(x)\right| \leq C+\frac{C}{\varepsilon}\left[\mathrm{e}^{-\frac{\sqrt{\alpha} x}{\varepsilon}}+\mathrm{e}^{\frac{-\sqrt{\alpha}(1-x)}{\varepsilon}}\right] \\
& \leq C\left\{1+\frac{1}{\varepsilon}\left[\mathrm{e}^{-\frac{\sqrt{\alpha x}}{\varepsilon}}+\mathrm{e}^{-\frac{-\sqrt{\alpha}(1-x)}{\varepsilon}}\right]\right\}
\end{aligned}
$$

which leads to the Equation (8).

\section{Discretizaton and Non-Uniform Mesh}

Let us consider the following any non-uniform mesh on $[0,1]$,

$$
\bar{\omega}_{N}=\left\{0=x_{0}<x_{1}<\cdots<x_{N-1}<x_{N}=1, i \geq 1, h_{i}=x_{i}-x_{i-1}, x_{N_{i}}=s_{i}, N_{i}=\frac{s_{i} N}{1}\right\} .
$$

We present some properties of the mesh function $g(x)$ defined on $\bar{\omega}_{N}$, which is needed in this section for analysis of the numerical solution.

$$
\begin{gathered}
g_{i}=g\left(x_{i}\right) \\
g_{x, i}=\frac{g_{i+1}-g_{i}}{h_{i+1}}, \quad g_{\bar{x}, i}=\frac{g_{i}-g_{i-1}}{h_{i}} \\
g_{\hat{x}, i}=\frac{g_{i+1}-g_{i}}{\hbar_{i}} \\
g_{\bar{x}, i}=\frac{g_{x, i}-g_{\bar{x}, i}}{\hbar_{i}}=\frac{1}{\hbar_{i}}\left(\frac{g_{i+1}-g_{i}}{h_{i+1}}-\frac{g_{i}-g_{i-1}}{h_{i}}\right) \\
h_{i}=x_{i}-x_{i-1}, \quad \hbar_{i}=\frac{h_{i}+h_{i+1}}{2} \\
\|g\|_{\infty}=\|g\|_{\infty, \bar{\omega}_{N}}=\max _{0 \leq i \leq N}\left|g_{i}\right| .
\end{gathered}
$$

Now, We will construct the difference scheme for the Equation (1). First, we integrate the Equation (1) over $\left(x_{i-1}, x_{i+1}\right)$,

$$
\hbar_{i}^{-1} \int_{x_{i-1}}^{x_{i+1}}-\varepsilon^{2} u^{\prime \prime}(x) \varphi_{i}(x) \mathrm{d} x+\hbar_{i}^{-1} \int_{x_{i-1}}^{x_{i+1}} f(x, u) \varphi_{i}(x) \mathrm{d} x=0, i=\overline{1, N-1}
$$

where $\left\{\varphi_{i}(x)\right\}, i=\overline{1, N-1}$ are the linear basis functions and having the form

$$
\varphi_{i}(x)= \begin{cases}\varphi_{i}^{(1)}(x)=\frac{x-x_{i-1}}{h_{i}}, & x_{i-1}<x<x_{i}, \\ \varphi_{i}^{(2)}(x)=\frac{x_{i+1}-x}{h_{i+1}}, & x_{i}<x<x_{i+1}, \\ 0, & x \notin\left(x_{i-1}, x_{i+1}\right)\end{cases}
$$

$\varphi_{i}^{(1)}(x)$ and $\varphi_{i}^{(2)}(x)$ are the solutions of the following problems,

$$
-\varepsilon^{2} \varphi^{\prime \prime}=0
$$




$$
\begin{gathered}
\varphi\left(x_{i-1}\right)=0, \varphi\left(x_{i}\right)=1 \\
-\varepsilon^{2} \varphi^{\prime \prime}=0 \\
\varphi\left(x_{i}\right)=1, \varphi\left(x_{i+1}\right)=0 .
\end{gathered}
$$

If we rearrange the Equation (31) it gives

$$
-\varepsilon^{2} \hbar_{i}^{-1} \int_{x_{i-1}}^{x_{i+1}} u^{\prime \prime}(x) \varphi_{i}(x) \mathrm{d} x+\hbar_{i}^{-1} \int_{x_{i-1}}^{x_{i+1}} f(x, u) \varphi_{i}(x) \mathrm{d} x=0, \quad i=\overline{1, N-1}
$$

After doing some calculation

$$
\varepsilon^{2} \hbar_{i}^{-1} \int_{x_{i-1}}^{x_{i+1}} u^{\prime}(x) \varphi_{i}^{\prime}(x) \mathrm{d} x+f\left(x_{i}, u_{i}\right)+R_{i}=0, \quad i=\overline{1, N-1}
$$

where

$$
R_{i}=\hbar_{i}^{-1} \int_{x_{i-1}}^{x_{i+1}} \mathrm{~d} x \varphi_{i}(x) \int_{x_{i-1}}^{x_{i+1}} \frac{\mathrm{d}}{\mathrm{d} x} f(x, u) K_{0}^{*}(x, \xi) \mathrm{d} \xi, \quad i=\overline{1, N-1}
$$

and

$$
K_{0}^{*}(x, \xi)=T_{0}(x-\xi)-T_{0}\left(\frac{x_{i+1}-x_{i-1}}{2}-\xi\right)+\left(x_{i+1}-x_{i-1}\right)^{-1}\left(x_{i+1}-\xi\right)^{0}\left(\frac{x_{i+1}-x_{i-1}}{2}-x\right) .
$$

So, from the Equation (37), the difference scheme is defined by

$$
-\varepsilon^{2} u_{\bar{x} \hat{x}, i}+f\left(x_{i}, u_{i}\right)+R_{i}=0, i=\overline{1, N-1} .
$$

Now, we define an approximation for the second boundary condition of the Equation (1). We accepted that $x_{N_{i}}$ is the mesh point nearest to $s_{i}$.

$$
\begin{aligned}
k_{0} u_{N} & =\sum_{i=1}^{m} k_{i} u_{N_{i}}+k_{m+1} \int_{0}^{1} u(x) \mathrm{d} x+d \\
& =\sum_{i=1}^{m} k_{i} u_{N_{i}}+k_{m+1}\left[\sum_{i=1}^{N} h_{i} u_{i}+r_{i}\right]+d
\end{aligned}
$$

where remainder term

$$
r_{i}=\sum_{i=1}^{N} \int_{x_{i-1}}^{x_{i}}\left(\xi-x_{i-1}\right) \frac{\mathrm{d}}{\mathrm{d} x} u(\xi) \mathrm{d} \xi
$$

By neglecting $R_{i}, r_{i}$ in the Equation (39) and the Equation (40), we suggest the following difference scheme for approximating the Equations (1)-(3)

$$
\begin{gathered}
-\varepsilon^{2} y_{\bar{x}, i}+f\left(x_{i}, y_{i}\right)=0, i=\overline{1, N} \\
y_{0}=0 \\
k_{0} y_{N}=\sum_{i=1}^{m} k_{i} y_{N_{i}}+k_{m+1} \sum_{i=1}^{N} h_{i} y_{i}+d .
\end{gathered}
$$

We will use the Shishkin mesh to be $\varepsilon$-uniform convergent of the difference scheme the Equations (42)-(44). So the Shishkin mesh divides each of the interval $[0, \sigma]$ and $[\sigma, 1-\sigma]$ into $N / 4$ equidistant subintervals and $[1-\sigma, 1]$ into $N / 2$ equidistant subinterval, where $\sigma$ and $1-\sigma$ are transition points which are defined as

$$
\sigma=\min \left\{\frac{1}{4},(\sqrt{\alpha})^{-1} \varepsilon \ln N\right\}
$$

if $h^{(1)}, h^{(2)}$ and $h^{(3)}$, respectively, are the stepsize on $[0, \sigma],[\sigma, 1-\sigma]$ and $[1-\sigma, 1]$. We have as 


$$
\begin{gathered}
h^{(1)}=\frac{4 \sigma}{N}, h^{(2)}=\frac{2(1-2 \sigma)}{N}, h^{(3)}=\frac{4 \sigma}{N}, \\
\frac{\left(h^{(1)}+h^{(3)}\right)}{2}=\frac{2}{N}, h^{(1)}=h^{(3)} \leq N^{-1}, N^{-1} \leq h^{(2)} \leq 2 N^{-1} \\
\bar{\omega}_{N}=\left\{x_{i}=i h^{(1)}, i=0, \frac{N}{4}, h^{(1)}=\frac{4 \sigma}{N} ; x_{i}=\sigma+\left(i-\frac{N}{4}\right) h^{(2)}, i=\frac{\bar{N}}{4}+1, \frac{3 N}{4},\right. \\
\left.h^{(2)}=\frac{2(1-2 \sigma)}{N} ; x_{i}=1-\sigma+\left(i-\frac{3 N}{4}\right) h^{(3)}, i=\frac{3 N}{4}+1, N, h^{(3)}=\frac{4 \sigma}{N}\right\}
\end{gathered}
$$

where, $N$ is even number, $\sigma \ll 1$.

\section{Error Analysis}

Let $z=y-u, \quad x \in \bar{w}_{N}$, which is the error function of the difference scheme the Equations (42), (43) and the solution of the discrete problem

$$
\begin{gathered}
l z \equiv-\varepsilon^{2} z_{\bar{x} \hat{x}, i}+\left[f\left(x_{i}, y_{i}\right)-f\left(x_{i}, u_{i}\right)\right]=R_{i}, \quad i=\overline{1, N-1} \\
z_{0}=0 \\
k_{0} z_{N}=\sum_{i=1}^{m} k_{i} z_{N_{i}}+k_{m+1} \sum_{i=1}^{N} h_{i} z_{i}=r_{i}, i=\overline{1, N}
\end{gathered}
$$

where $R_{i}, r_{i}$ are defined in the Equation (38) and the Equation (41).

Lemma 4.2. Let $z_{i}$ be the solution of the Equations (45)-(47) and approximation error $R_{i}$ and $r_{i}$. Then there are the following inequalities,

$$
\begin{gathered}
\|R\|_{\infty, \bar{w}_{N}} \leq C N^{-1} \ln N \\
|r|_{\infty, \bar{w}_{N}} \leq C N^{-1} \ln N
\end{gathered}
$$

Proof. We evaluate the Equation (38) and the Equation (41), respectively

$$
\begin{gathered}
R_{i}=\hbar_{i}^{-1} \int_{x_{i-1}}^{x_{i+1}} \mathrm{~d} x \varphi_{i}(x) \int_{x_{i-1}}^{x_{i+1}} \frac{\mathrm{d}}{\mathrm{d} x} f(x, u) K_{0}^{*}(x, \xi) \mathrm{d} \xi \\
\left|R_{i}\right| \leq \hbar_{i}^{-1} \int_{x_{i-1}}^{x_{i+1}} \varphi_{i}(x) \mathrm{d} x \int_{x_{i-1}}^{x_{i+1}}\left|\frac{\partial f(\xi, u(\xi))}{\partial \xi}+\frac{\partial f}{\partial u} \frac{\mathrm{d} u(\xi)}{\mathrm{d} \xi}\right| \mathrm{d} \xi \leq C \int_{x_{i-1}}^{x_{i+1}}\left(1+u^{\prime}(\xi)\right) \mathrm{d} \xi .
\end{gathered}
$$

Consequently,

$$
\left|R_{i}\right| \leq C\left\{\int_{x_{i-1}}^{x_{i+1}}\left(1+\frac{1}{\varepsilon}\left[\mathrm{e}^{\frac{-\sqrt{\alpha} x}{\varepsilon}}+\mathrm{e}^{\frac{-\sqrt{\alpha}(1-x)}{\varepsilon}}\right]\right) \mathrm{d} x\right\} .
$$

In the beginning, we consider the case $\sigma=1-\sigma=\frac{1}{4}$ and so $x_{i} \in[0, \sigma]$,

$$
\frac{1}{4}<(\sqrt{\alpha})^{-1} \varepsilon \ln N, h=h^{(1)}=h^{(2)}=h^{(3)}=\frac{1}{N}
$$

it then follows from the Equation (50) that

$$
\left|R_{i}\right| \leq C\left\{h+\frac{1}{\varepsilon} \int_{x_{i-1}}^{x_{i+1}} \mathrm{~d} x\right\} \leq C\left\{h+\varepsilon^{-1} h\right\} \leq C N^{-1} \ln N, 1<i<N .
$$


Second, we consider the case $\sigma=(\sqrt{\alpha})^{-1} \varepsilon \ln N$, and so $\frac{1}{4}>(\sqrt{\alpha})^{-1} \varepsilon \ln N$, we estimate $R_{i}$ on $[0, \sigma],[\sigma, 1-\sigma]$ and $[1-\sigma, 1]$, respectively.

In the seperate $[0, \sigma]$, the Equation (50) reduces to

$$
\left|R_{i}\right| \leq C\left(1+\varepsilon^{-1}\right) h^{(1)} \leq C\left(1+\varepsilon^{-1}\right) \frac{4 \sigma}{N} \leq C N^{-1} \ln N, 1 \leq i<\frac{N}{4}-1 .
$$

In the seperate $[\sigma, 1-\sigma]$, the Equation (50) reduces to

$$
\begin{aligned}
\left|R_{i}\right| & \leq C\left\{h^{(2)}+(\sqrt{\alpha})^{-1}\left[\left(\mathrm{e}^{\frac{-\sqrt{\alpha} x_{i-1}}{\varepsilon}}-\mathrm{e}^{\frac{-\sqrt{\alpha} x_{i+1}}{\varepsilon}}\right)+\left(\mathrm{e}^{\frac{-\sqrt{\alpha}\left(1-x_{i+1}\right)}{\varepsilon}}-\mathrm{e}^{\left.\left.\left.\frac{-\sqrt{\alpha}\left(1-x_{i-1}\right)}{\varepsilon}\right)\right]\right\}}\right.\right.\right. \\
& \leq C\left(1+\varepsilon^{-1}\right) \frac{4 \sigma}{N} \leq C N^{-1} \ln N, \quad 1 \leq i<\frac{N}{4}-1
\end{aligned}
$$

where for $x_{i}=(\sqrt{\alpha})^{-1} \varepsilon \ln N+\left(i-\frac{N}{4}\right) h^{(2)}$,

$$
x_{i-1}=(\sqrt{\alpha})^{-1} \varepsilon \ln N+\left(i-1-\frac{N}{4}\right) h^{(2)}, x_{i+1}=(\sqrt{\alpha})^{-1} \varepsilon \ln N+\left(i+1-\frac{N}{4}\right) h^{(2)}
$$

and so

$$
\mathrm{e}^{\frac{-\sqrt{\alpha} x_{i-1}}{\varepsilon}}-\mathrm{e}^{\frac{-\sqrt{\alpha} x_{i+1}}{\varepsilon}} \leq N^{-1}
$$

Analogously for $x_{i}=1-\sigma+\left(i-\frac{3 N}{4}\right) h^{(3)}$,

$$
x_{i-1}=1-\sigma+\left(i-1-\frac{3 N}{4}\right) h^{(3)}, x_{i+1}=1-\sigma+\left(i+1-\frac{3 N}{4}\right) h^{(3)}
$$

thus

$$
\mathrm{e}^{\frac{-\sqrt{\alpha}\left(1-x_{i+1}\right)}{\varepsilon}}-\mathrm{e}^{\frac{-\sqrt{\alpha}\left(1-x_{i-1}\right)}{\varepsilon}} \leq N^{-1}
$$

according to the Equation (54) and the Equation (55), we can rewrite the the Equation (53)

$$
\left|R_{i}\right| \leq C\left\{h^{(2)}+(\sqrt{\alpha})^{-1}\left[N^{-1}+N^{-1}\right]\right\} \leq C N^{-1} .
$$

In the seperate $[1-\sigma, 1]$, the Equation (50) reduces to

$$
\left|R_{i}\right| \leq C\left(1+\varepsilon^{-1}\right) h^{(3)} \leq C\left(1+\varepsilon^{-1}\right) \frac{4 \sigma}{N} \leq C N^{-1} \ln N, \frac{3 N}{4}+1 \leq i \leq N .
$$

The last estimate is for $x_{N / 4}$ and $x_{3 N / 4}$ :

We rewrite the the Equation (50) for $i=\frac{N}{4}$,

$$
\left|R_{\frac{N}{4}}\right| \leq C\left\{\int_{\frac{x_{N}}{4}}^{\frac{x_{N}}{4}+1}\left(1+\frac{1}{\varepsilon}\left[\mathrm{e}^{\frac{-\sqrt{\alpha} x}{\varepsilon}}+\mathrm{e}^{\frac{-\sqrt{\alpha}(1-x)}{\varepsilon}}\right]\right) \mathrm{d} x\right\} .
$$

We take integrate in the Equation (58) and so

$$
\left|R_{\frac{N}{4}}\right| \leq C\left\{\left(x_{\frac{N}{4}+1}-x_{\frac{N}{4}}-\frac{1}{\varepsilon} \frac{\varepsilon}{\sqrt{\alpha}}\left[\left(\mathrm{e}^{\frac{-\sqrt{\alpha} x_{\frac{N}{4}}+1}{\varepsilon}}-\mathrm{e}^{\frac{-\sqrt{\alpha} x_{\frac{N}{4}}}{\varepsilon}}\right)+\left(\mathrm{e}^{\frac{-\sqrt{\alpha}\left(1-x_{\frac{N}{N}}\right)}{\varepsilon}}-\mathrm{e}^{\left.\frac{-\sqrt{\alpha}\left(1-\chi_{N}\right.}{4}\right)} \varepsilon\right)\right]\right\}\right.
$$


where

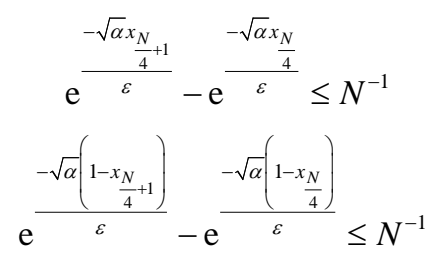

we rewrite the Equation (59) with the Equation (60) and the Equation (61), thus,

$$
\left|R_{\frac{N}{4}}\right| \leq C\left\{x_{\frac{N}{4}+1}-x_{\frac{N}{4}}+2 N^{-1}\right\} \leq C\left\{h^{(2)}+N^{-1}\right\} \leq C N^{-1} \ln N
$$

where $h^{(2)}=2\left(1-(\sqrt{\alpha})^{-1} \varepsilon \ln N\right)$.

We use in a similar way as above for $i=\frac{3 N}{4}$, and so

$$
\left|\frac{R_{3 N}}{4}\right| \leq C N^{-1} \ln N .
$$

Next, we use estimate for the remainder term $r$ :

From the Equation (41) we can write

$$
\left|r_{i}\right| \leq \sum_{i=1}^{N} \int_{x_{i-1}}^{x_{i}}\left(x-x_{i-1}\right) \frac{\mathrm{d}}{\mathrm{d} x} u(x) \mathrm{d} x \leq \sum_{i=1}^{N} h_{i} \int_{x_{i-1}}^{x_{i}} u^{\prime}(x) \mathrm{d} x
$$

from the Equation (8)

$$
\begin{aligned}
& \left|r_{i}\right| \leq \sum_{i=1}^{N} h_{i} \int_{x_{i-1}}^{x_{i}} C\left\{1+\frac{1}{\varepsilon}\left[\mathrm{e}^{\frac{-\sqrt{\alpha} x}{\varepsilon}}+\mathrm{e}^{\frac{-\sqrt{\alpha}(1-x)}{\varepsilon}}\right]\right\} \mathrm{d} x, 1 \leq i \leq N \\
& \left|r_{i}\right| \leq \sum_{i=1}^{N / 4} h^{(1)} \int_{x_{i-1}}^{x_{i}}\left(1+\frac{1}{\varepsilon}\left[\mathrm{e}^{\frac{-\sqrt{\alpha} x}{\varepsilon}}+\mathrm{e}^{\frac{-\sqrt{\alpha}(1-x)}{\varepsilon}}\right]\right) \mathrm{d} x+\sum_{i=N / 4+1}^{3 N / 4} h^{(2)} \int_{x_{i-1}}^{x_{i}}\left(1+\frac{1}{\varepsilon}\left[\mathrm{e}^{\frac{-\sqrt{\alpha} x}{\varepsilon}}+\mathrm{e}^{\frac{-\sqrt{\alpha}(1-x)}{\varepsilon}}\right]\right) \mathrm{d} x \\
& +\sum_{i=3 N / 4+1}^{N} h^{(3)} \int_{x_{i-1}}^{x_{i}}\left(1+\frac{1}{\varepsilon}\left[\mathrm{e}^{\frac{-\sqrt{\alpha} x}{\varepsilon}}+\mathrm{e}^{\frac{-\sqrt{\alpha}(1-x)}{\varepsilon}}\right]\right) \mathrm{d} x \\
& =h^{(1)} \int_{0}^{\sigma}\left(1+\frac{1}{\varepsilon}\right) \mathrm{d} x+h^{(2)} \int_{\sigma}^{1-\sigma}\left(1+\frac{1}{\varepsilon}\right) \mathrm{d} x+h^{(3)} \int_{1-\sigma}^{1}\left(1+\frac{1}{\varepsilon}\right) \mathrm{d} x \\
& \left|r_{i}\right| \leq C\left[h^{(1)}+h^{(2)}+h^{(3)}\right] \leq C\left[N^{-1}+2 N^{-1}+N^{-1}\right] \leq C N^{-1}
\end{aligned}
$$

Lemma 4.3. Let $z_{i}$ be solution of the Equations (45)-(47). Then there is the following inequality,

$$
\|z\|_{\infty, \bar{w}_{N}} \leq C\left[\|R\|_{\infty, w_{N}}+|r|\right] .
$$

Proof. Rearranging the Equation (45) gives

$$
l z \equiv-\varepsilon^{2} z_{\vec{x} x, i}+a_{i} z_{i}=R_{i}, \quad i=\overline{1, N-1}
$$

where

$$
a_{i}=\frac{\partial F}{\partial u}\left(t_{i}, u_{i}+\gamma z_{i}\right), 0<\gamma<1
$$

according to the proof of Lemma 2.1, we can use the maximum principle, and so it is easy to obtain, 


$$
\begin{aligned}
z_{\infty, \bar{w}_{N}} & \leq\left|z_{N}\right|+\alpha^{-1}\left(\|R\|_{\infty, w_{N}}+|r|\right) \\
& \leq\left|\sum_{i=1}^{m} k_{i} z_{N_{i}}+k_{m+1} \sum_{i=1}^{N} h_{i} z_{i}+d-r_{i}\right|+\alpha^{-1}\left(\|R\|_{\infty, w_{N}}+|r|\right) \\
& \leq \alpha^{-1}\left(\|R\|_{\infty, w_{N}}+|r|\right) \leq C\left[\|R\|_{\infty, w_{N}}+|r|\right]
\end{aligned}
$$

Conclusion 4.1. We know that the solution of the Equations (1)-(3) is $u(x)$ and the solution of the Equations (45)-(47) is $y_{i}$. Then Lemma (4.2) and Lemma (4.3) give us the following convegence result

$$
\|y-u\|_{\infty, \bar{w}_{N}} \leq C N^{-1} \ln N \text {. }
$$

\section{Numerical Example}

In this section, an example of nonlinear singularly perturbed multi-point boundary value problem is given to illustrate the efficiency of the numerical method described above. The example is computed using maple 10. Results obtained by the method are compared with the exact solution of example and found to be good agreement with each other. We compute approximate errors $e_{\varepsilon}^{N}, e^{N}$ and the convergence rates $p_{\varepsilon}^{N}$ on the Shishkin Mesh $\bar{\omega}_{N}$ for different values of $\varepsilon, N$.

Example 5.1.

We solve the difference scheme the Equations (42), (44) using the following iteration technique,

$$
\begin{gathered}
{\left[\frac{\varepsilon^{2}}{\hbar_{i} h_{i}}\right] y_{i-1}^{(n)}-\left[\frac{2 \varepsilon^{2}}{h_{i} h_{i+1}}+\frac{\partial f}{\partial y}\left(x_{i}, y_{i}^{(n-1)}\right)\right] y_{i}^{(n)}+\left[\frac{\varepsilon^{2}}{\hbar_{i} h_{i+1}}\right] y_{i+1}^{(n)}} \\
=f\left(x_{i}\right)+f\left(x_{i}, y_{i}^{(n-1)}\right)-y_{i}^{(n-1)} \frac{\partial f}{\partial y}\left(x_{i}, y_{i}^{(n-1)}\right), i=1, \cdots, N-1, \quad n=1,2, \cdots \\
y_{0}^{(n)}=0, k_{0} y_{N}^{(n)}=\sum_{i=1}^{m} k_{i} y_{N_{i}}^{(n-1)}+k_{m+1} \sum_{i=1}^{N} h_{i} y_{i}^{(n-1)}+d \\
{\left[\frac{\varepsilon^{2}}{\hbar_{i} h_{i}}\right] y_{i-1}^{(n)}-\left[\frac{2 \varepsilon^{2}}{h_{i} h_{i+1}}+\frac{\partial f}{\partial y}\left(x_{i}, y_{i}^{(n-1)}\right)\right] y_{i}^{(n)}+\left[\frac{\varepsilon^{2}}{\hbar_{i} h_{i+1}}\right] y_{i+1}^{(n)}} \\
=f\left(x_{i}\right)+f\left(x_{i}, y_{i}^{(n-1)}\right)-y_{i}^{(n-1)} \frac{\partial f}{\partial y}\left(x_{i}, y_{i}^{(n-1)}\right), \quad i=1, \cdots, N-1, \\
y_{\frac{N}{2}}=\mu_{n-1}, y_{N}=\mu_{n-1}-1
\end{gathered}
$$

where

$$
\begin{gathered}
\mu_{n}=\frac{\left(\frac{\varepsilon}{h_{2}}\right)^{2} y_{\frac{N}{2}-1, n}+\left(\frac{\varepsilon}{h_{2}}\right)^{2} y_{\frac{N}{2}+1, n}-1+\left(y_{\frac{N}{2}+1, n-1}\right)^{2}}{2\left(\frac{\varepsilon}{h_{2}}\right)^{2}+y_{\frac{N}{2}, n-1}} \\
y_{\frac{N}{2}}=\mu_{n}, \quad \mu_{0}=C_{0} \geq 1 .
\end{gathered}
$$

The system of the Equations (68)-(70) is solved by the following procedure,

$$
\begin{gathered}
A_{i}=\frac{\varepsilon^{2}}{\hbar_{i} h_{i}}, B_{i}=\frac{\varepsilon^{2}}{\hbar_{i} h_{i+1}}, C_{i}=\frac{2 \varepsilon^{2}}{h_{i} h_{i+1}}+\frac{\partial f}{\partial y}\left(x_{i}, y_{i}^{(n-1)}\right), \\
F_{i}=-f\left(x_{i}, y_{i}^{(n-1)}\right)+y_{i}^{(n-1)} \frac{\partial f}{\partial y}\left(x_{i}, y_{i}^{(n-1)}\right)
\end{gathered}
$$




$$
\begin{gathered}
\alpha_{1}=0, \beta_{1}=0 \\
\alpha_{\frac{N}{2}+1}=0, \beta_{\frac{N}{2}+1}=\mu_{n-1} \\
\alpha_{i+1}=\frac{B_{i}}{C_{i}-A_{i} \alpha_{i}}, \beta_{i+1}=\frac{F_{i}+A_{i} \beta_{i}}{C_{i}-A_{i} \alpha_{i}}, i=1, \cdots, N-1 \\
y_{i}^{(n)}=\alpha_{i+1} y_{i+1}^{(n)}+\beta_{i+1}, y_{i}^{(0)}=0.5, i=N-1, \cdots, 2,1 .
\end{gathered}
$$

It is easy to verify that

$$
A_{i}>0, B_{i}>0, C_{i}>A_{i}+B_{i}, i=1, \cdots, N .
$$

For this reason, the described procedure above is stable. Also, the Equations (42)-(44) has only one solution.

Now, we consider the following test problem,

$$
\begin{gathered}
-\varepsilon^{2} u^{\prime \prime}+u^{2}(x)-f(x)=0, \\
u(0)=0 \\
u(1)=u(0.5)+d
\end{gathered}
$$

which has the exact solution,

$$
u(x)=\frac{(2 x-1)\left(\mathrm{e}^{\frac{1-x}{\varepsilon}}-\mathrm{e}^{\frac{x}{\varepsilon}}\right)}{\mathrm{e}^{\frac{1}{\varepsilon}}-1}+1
$$

In the computations in this section, we will take $\alpha=2, d=-1$ the initial guess in the iteration procedure is $y_{i}^{(0)}=0.5$. The stopping criterion is taken as

$$
\max _{i}\left|y_{i}^{(n+1)}-y_{i}^{(n)}\right| \leq 10^{-5} .
$$

The error estimates are denoted by

$$
e_{\varepsilon}^{N}=\|y-u\|_{\infty, \bar{\omega}_{N}}
$$

and

$$
e^{N}=\max _{\varepsilon} e_{\varepsilon}^{N} .
$$

The convergence rates are

$$
P_{\varepsilon}^{N}=\log _{2}\left(\frac{e^{N}}{e^{2 N}}\right) .
$$

The numerical results obtained from the problem of the difference scheme by comparison, the error and uniform rates of convergence were found and these are shown in Table 1. Consequently, numerical results show that the proposed scheme is working very well.

The results point out that the convergence rate of the established scheme is really in unision with theoretical analysis.

From the graps it is show that the error is maximum near the boundary layer and it is almost zero in outer region in the Figure 3. Approximate solution compared with exact solution in Figure 2. Approximate solutions are given for different values of $\varepsilon$ in Figure 1 . 
Table 1. The computed maximum pointwise errors $e^{N}$ and $e^{2 N}$, the numerical rate of convergence $p^{N}$ on the Shishkin mesh $\bar{\omega}_{N}$ for different values of $N$ and $\varepsilon$.

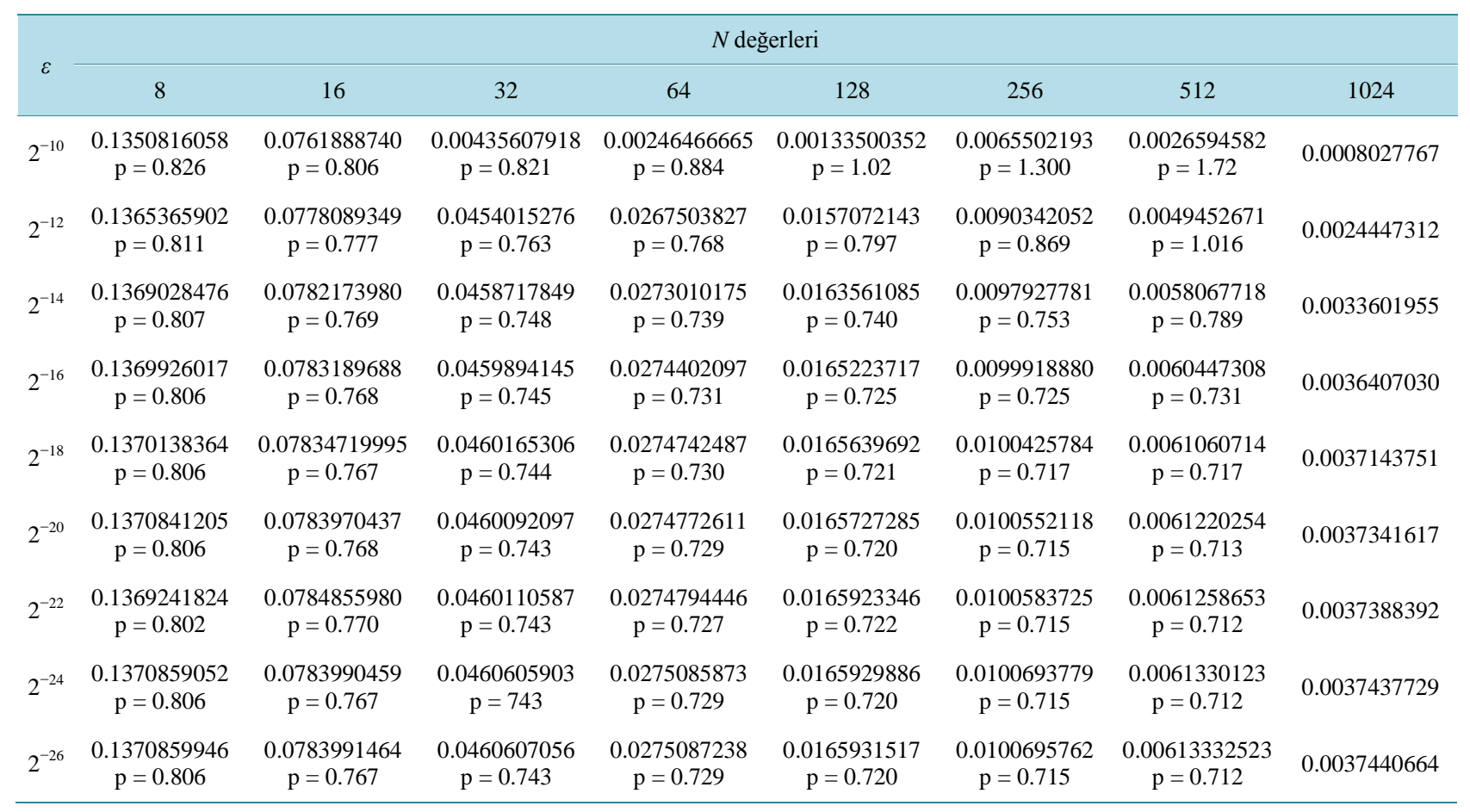

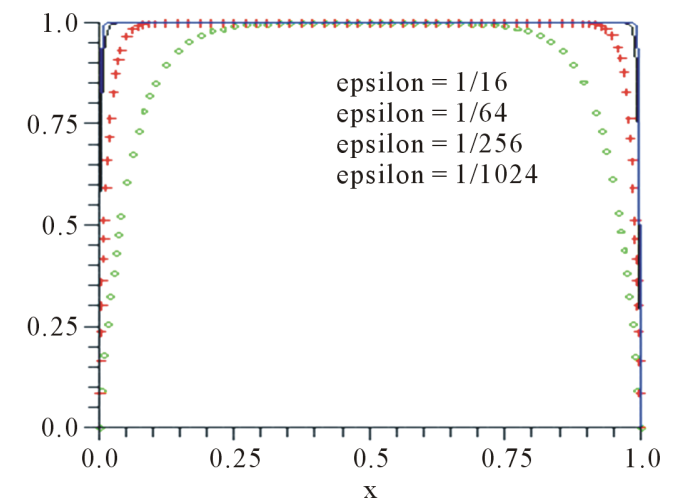

Figure 1. Approximate solution distribution for $\varepsilon=2^{-4}, 2^{-6}, 2^{-8}, 2^{-10}$ using $N=256$.

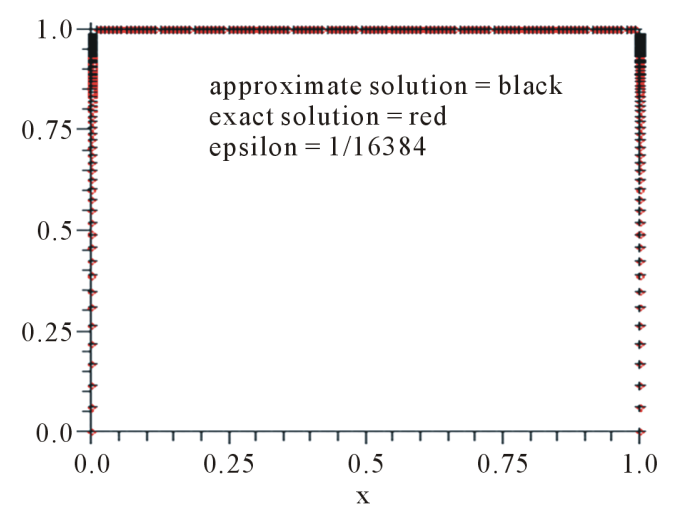

Figure 2. Comparison of approximate solution and exact solution for $\varepsilon=2^{-14}$. 


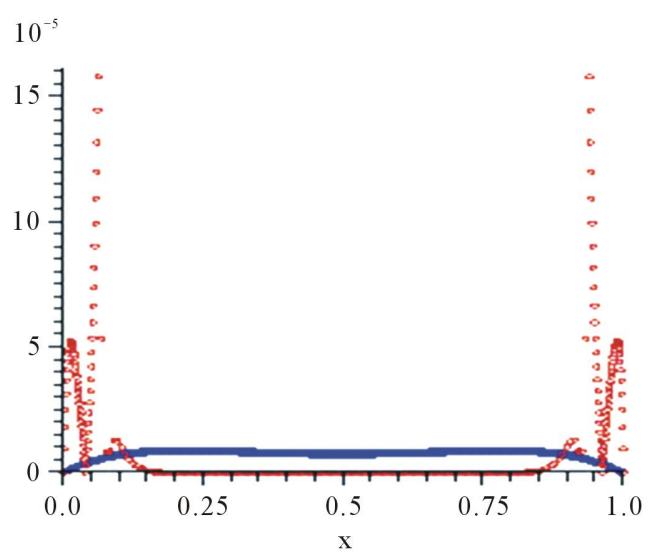

Figure 3. Error distribution for $\varepsilon=2^{-2}, 2^{-8}, 2^{-10}, 2^{-12}$ using $N=256$.

\section{Conclusion}

Consequently, the aim of this paper was to give uniform finite difference method for numerical solution of nonlinear singularly perturbed problem with nonlocal boundary conditions. The numerical method was constructed on Shishkin mesh. The method was pointed out to be convergent, uniformly in the $\varepsilon$-parameter, of first order in the discrete maximum norm. The numerical example illustrated in practice the result of convergence proved theoretically.

\section{References}

[1] Cziegis, R. (1988) The Nümerical Solution of Singularly Pertürbed Nonlocal Problem. Lietuvos Matematikos Rinkinys, 28, 144-152. (In Russian)

[2] Cziegis, R. (1991) The Difference Schemes for Problems with Nonlocal Conditions. Informatica (Lietuva), 2, $155-170$.

[3] Bakhvalov, N.S. (1969) On Optimization of Methods for Solving Boundary-Value Problems in the Presence of a Boundary Layer. The Use of Special Transformations in the Numerical Solution of Bounary-Layer Problems. Zhurnal Vychislitel'noi Matematiki i Matematicheskoi Fiziki, 9, 841-859.

[4] Amiraliyev, G.M. and Cakir, M. (2007) Numerical Solution of a Singularly Perturbed Three-Point Boundary Value Problem. International Journal of Applied Mathematics, 84, 1465-1481.

[5] Amiraliyev, G.M. and Çakır, M. (2000) A Uniformily Convergent Difference Scheme for Singularly Perturbed Problem with Convective Term End Zeroth Order Reduced Equation. International Journal of Applied Mathematics, 2, 1407-1419.

[6] Amiraliyev, G. M. and Çakır, M. (2002) Numerical Solution of the Singularly Perturbed Problem with Nonlocal Boundary Condition. Applied Mathematics and Mechanics (English Edition), 23, 755-764. http://dx.doi.org/10.1007/BF02456971

[7] Amiraliyev, G.M. and Duru, H. (2005) A Note on a Parameterized Singular Perturbation Problem. Journal of Computational and Applied Mathematics, 182, 233-242. http://dx.doi.org/10.1016/j.cam.2004.11.047

[8] Amiraliyev, G.M. and Erdoğan, F. (2007) Uniform Numerical Method for Singularly Perturbed Delay Differential Equations. Computers \& Mathematics with Applications, 53, 1251-1259. http://dx.doi.org/10.1016/j.camwa.2006.07.009

[9] Amiraliyeva, I.G., Erdoğan, F. and Amiraliyev, G.M. (2010) A Uniform Numerical Method for Dealing with a Singularly Perturbed Delay İnitial Value Problem. Applied Mathematics Letters, 23, 1221-1225. http://dx.doi.org/10.1016/j.aml.2010.06.002

[10] Adzic, N. and Ovcin, Z. (2001) Nonlinear Spp with Nonlocal Boundary Conditions and Spectral Approximation. Novi Sad Journal of Mathematics, 31, 85-91.

[11] Amiraliyev, G.M., Amiraliyeva, I.G. and Kudu, M. (2007) A Numerical Treatment for Singularly Perturbed Differential Equations with İntegral Boundary Condition. Applied Mathematics and Computations, 185, 574-582. http://dx.doi.org/10.1016/j.amc.2006.07.060

[12] Herceg, D. (1990) On the Numerical Solution of a Singularly Perturbed Nonlocal Problem. Univ. u Novom Sadu Zb. Rad. Prirod.-Mat. Fak. Ser. Mat., 20, 1-10. 
[13] Herceg, D. (1991) Solving a Nonlocal Singularly Perturbed Problem by Splines in Tension. Univ. u Novom Sadu Zb. Rad. Prirod.-Mat. Fak. Ser. Mat, 21, 119-132.

[14] Çakır, M. (2010) Uniform Second-Order Difference Method for a Singularly Perturbed Three-Point Boundary Value Problem. Hindawi Publising Corporation Advances in Difference Equations, Vol. 2010, 13 p.

[15] Geng, F.Z. (2012) A Numerical Algorithm for Nonlinear Multi-Point Boundary Value Problems. Journal of Computational and Applied Mathematics, 236, 1789-1794. http://dx.doi.org/10.1016/j.cam.2011.10.010

\section{Submit or recommend next manuscript to SCIRP and we will provide best service for you:}

Accepting pre-submission inquiries through Email, Facebook, Linkedin, Twitter, etc A wide selection of journals (inclusive of 9 subjects, more than 200 journals)

Providing a 24-hour high-quality service

User-friendly online submission system

Fair and swift peer-review system

Efficient typesetting and proofreading procedure

Display of the result of downloads and visits, as well as the number of cited articles

Maximum dissemination of your research work

Submit your manuscript at: http://papersubmission.scirp.org/ 